\title{
XIII.
}

Aus der K. K. chirurg. Klinik des Herrn Hofrathes Prof. Nicoladoni in Graz.

\section{Beiträge zur Technik einiger Operationen am Dickdarme.}

\author{
Von \\ Dr. Erwin Payr, \\ Privatdocent für Chirorgie und Assistent obiger Elinik.
}

(Mit 5 Abbildungen.)

Nachfolgende Zeilen beziehen sich auf einige technische Fragen bei der Ausführung von Operationen am Dickdarm. Nicht das Aufstellen von "neuen Methoden", sondern die objective Darstellung dessen, was sich dem Einzelnen als zweckmässig erwiesen hat, ist der von uns beabsichtigte Endzweck derartiger Mittheilungen; oftmals sind sie für den Leser nur eine Bestätigung eigener gedachter und auch ausgeführter Vorgänge, manchmal erweisen sie sich für diese oder jene Situation als nützlich.

I. Zur Versorgung des centralen Darmendes nach der Resection oder Amputation des Mastdarmes.

Der letzte Chirurgen-Congress bot reichlich Gelegenheit, einen Ueberblick über den gegenwärtigen Stand der Technik, als auch unmittelbare und Dauerresultate bei den sacralen Operationen am Mastdarm zu gewinnen. - Das centrale Darmende wird, wenn es sich um eine Resectio recti handelt, entweder mit dem peripheren erhalten gebliebenen durch circuläre Naht vereinigt, - dies geschieht entweder in der sacralen Wunde, oder nach geschehener Invagination vor der Analöffnung - oder es wird das centrale Stïck durch den erhaltenen Sphincterantheil des rectums durchgezogen und in irgend einer Weise am anus befestigt.

Beide Methoden, die Circulär-Naht und die Durchzugsmethode kommen gegenwärtig in Verwendung. Mehr Sympathie findet der Vorgang des Durchziehens des centralen Darmstückes durch den stehen gebliebenen Analtheil, weil allen Nahtmethoden, 
die die beiden Darmquersehnitte circulär vereinigen, der Nachtheil anhaftet, dass durch Aufgehen der Naht Fistelbildungen entstehen. Auch die nach Invagination beider Darmantheile gemachte und dadurch viel bequemere Circulärnaht schützt davor nicht (Wölfler). 1)

$\mathrm{Hoch}$ enegg, ${ }^{2}$ ) dem ein hervorragendes Verdienst um den Entwicklungsgang der sacralen Operationen unbedingt zugesprochen werden muss, hat bereits vor 11 Jahren die Durchzugsmethode ausgeführt; er zog den centralen Antheil des Darmes nach ausgeführter Resection durch den erhaltenen Analtheil, aus dem die Schleimhaut zuvor exstirpirt worden war.

$\mathrm{Kraske}^{3}$ ) hatte bereits in seiner ersten Mittheilung im Jahre 1885 von einem Invaginationsplan gesprochen, der vorerst noch complicirt war und später bedeutende Vereinfachung erfuhr und sich fast mit dem von Hochenegg (l. c.) ausgeführten Verfahren deckt.

Das centrale Darmlumen wurde an die Analportion angenäht; von der Wunde aus wurde eine einige Centim. höher gelegene Nahtreihe angelegt, die dem durchgezogenen Darme noch mehr Festigkeit verlieh. Dadurch werden breite Flächen aneinandergelegt, die der Schleimhaut entblösste Fläche des erhaltenen untersten RectumAbschnittes steht in innigem Contact mit der serosa-überkleideten Aussenfläche des durchgezogenen Darmes. Die Methode der circulären Darmnahtanlegung vor dem anus nach geschehener Invagination des centralen in den peripheren Darmantheil wurde ebenfalls von Hochenegg (l. c.) ausgeführt und theilte Wölfler (l. c.) sein gleichartiges Vorgehen mit.

Es sei hier erwähnt, dass Heinecke ${ }^{4}$ ) derjenige war, der das erste Verfahren der Versorgung des proximalen Darmendes bei der Resection mit Invagination in das periphere Stück angab.

1) Wölfler, Verhandlungen der Deutschen Gesellschaft f. Chirurgie. 1900. Discussion. I. Theil. S. $38-39$.

2) Hochenegg, Zur Therapie des Rectum carcinomes. Wiener klinische Wochenschrift. 1897. Nr. 32. - Wiener klin. Wochenschrift. 1888. Nr. 11-16. 1889. Nr. 26-30. - Bericht über 121 eigene sacrale Mastdarmoperationen wegen Carcinom u. s. w. Verhandlungen der Deutschen Gesellschaft f. Chirurgie. 1900. I. Theil. S. 14-31.

3) Kraske, Verhandlungen der Deutschen Gesellschaft f. Chirurgie. 1885. - Dic sacrale Methode der Exstirpation von Mastdarmkrebsen und die Resectio recti. Berliner kliu. Wochenschr. 1887. Nr.48. - Erfahrungen ö̈ber den Mastdarmkrebs. Sammlung klin. Vorträge v. Volkmann's. Neue Folge Nr. 183-184. 1897 .

4) v. Heinecke, Verhandlungen der chirurgischen Section der 65. Versammlung deutscher Naturforscher u. Aerzte. Centralbl. f. Chirurgie. 1893. Nr. 46. S. 1018 . 
Sowohl was die Reinlichkeit überhaupt und besonders die In fectionsgefahr der sacralen Wunde anlangt, ist das Durchzugs verfahren weitaus vorzuziehen. - Das einzige Bedenken, das man gegen dasselbe erheben könnte, ist, dass in manchen Fällen ein mehr oder minder grosses Stück des durchgezogenen Darmes abstirbt und die Möglichkeit besteht, dass sich der Darm durch die Analportion zurückzieht und durch sein offenes unversorgtes Ende die sacrale Wunde, sowie möglicherweise das Peritoneum inficirt.

Nach unseren Erfahrungen jedoch lässt sich dieses höchst unliebsame Ereigniss sicher vermeiden und hat Kümmell ${ }^{1}$ ) am ChirurgenCongress des Jahres 1899 auch die nöthigen Argumente beigebracht, die uns über die Ernährungsverhältnisse des Darmes nach Resection grösserer und kleinerer Stücke Aufschluss geben. Wir kommen auf diesen Punkt unten zurück.

Eine wesentliche Vereinfachung der sogenannten Durchzugsmethode wurde von Herrn Hofrath Nicoladoni 2), meinem hochverehrten Chef, im Jahre 1897 mitgetheilt. Das Verfahren besteht darin, dass der proximale Stumpf des Rectums nach ausgeführter Resection durch den After gezogen und durch einen $3-4 \mathrm{~cm}$ weiten Ring am Zurückschlüpfen verhindert wird. Der Ring besteht aus Silberdrath und ist mit Jodoformgaze umwickelt. Mit fortlaufender Naht wird das Darmstïck an diesen Ring befestigt. Aus dem distalen Darmstiicke wird die Schleimhaut exstirpirt; so sind breite für die Verheilung geeignete Flächen in Contact gebracht. Einige innere Nähte befestigen den durchgezogenen Dickdarmstumpf am proximalen Theile des stehengebliebenen Enddarmes. Das Verfahren ist rasch auszuführen und begünstigt die Reinheit der Wundverhältnisse. Es sollen nach der Resectio recti salinische Abführmittel gegeben werden, anstatt des früher gebräuchlichen Opiums. Es kommt durch dieses nur zu einer schädlichen Ansammlung von Kothmassen im Darme; die Anwendung von Klysmen ist durch das Ring-Verfahren sehr erleichtert.

Der grosse Vortheil des Verfahrens von Nicoladoni zeigte sich nicht nur an unseren klinischen Patienten, sondern auch von andrer Seite kamen Bestätigungen über die Zweckmässigkeit und die grossen

1) Kümmell, Ueber Resection des Colon descendens und Fixation des Colon transversum in den Analring. Verhandlungen der Deutschen Gesellschaft f. Chirurgie. 1899. II. Theil. S. $432 \mathrm{ff}$.

2) Nicoladoni, Zur Naht bei Resectio recti. Centralbl. f. Chirurgie. 1897. Nr. 35. 
Vorzüge dieser Technik. Sieber ${ }^{1}$ ) hat in einer I. Diss. Mittheilungen über 33 derartig operirte Fälle aus der Klinik v. Mikulicz's gemacht, und Henle ${ }^{2}$ ) betonte auf dem vorletzten Chirurgen-Congress die auffallende und erhebliche Verbesserung der Resultateder sacralen Rectum-Operationen seit der Verwendung des Nicoladoni'schen (l. c.) Verfahrens. Sowohl Wund-als Peritoneal-Infection wird durch dasselbe vermieden, die Darmentleerung frühzeitig ermöglicht, die Heilungsdauer verkïrzt, nicht nur durch die verbesserten Wundheilungsverhältnisse, sondern auch durch viel seltener gewordene Nachoperationen. Auch K ümmell (l. c.) hat bei seinen Rectum-Operationen das Verfahren in Anwendung gezogen.

Ich versuchte nun, anstatt des Silberdrahtringes zur Erreichung: des gleichen Zweckes einen besonders grossen für die Verhältnisse des Dickdarmes geformten Murphyknopf zu benützen, der nach Durchziehen des proximalen Darmstïckes durch den After so angelegt wird, dass der männliche Theil des Knopfes in die mit der gewöhnlichen Tabaksbeutelnaht armirte Oeffnung des centralen Darmstïckes eingeschoben wird und durch das Aufschieben des weiblichen Theiles und leichtes Aneinanderdrücken der Knopfhälften befestigt wird. Im männlichen Theile des Knopfes steckt ein dickes das Lumen des Knopfes vollkommen ausfüllendes reichlich mit Vaseline bestrichenes Gummidrainrohr, das den Knopf wasserdicht abschliesst, eine Länge von ca. ${ }^{1 / 4} \mathrm{~m}$ hat und ausserhalb des Verbandes in eine Vorlage mündet. Hat man Eile, so ist nicht einmal die Tabaksbeutelnaht am Ende des durchgezogenen Darmstückes nothwendig, sondern es genügt eine daselbst circulär angelegte feste Seidenligatur, die den Darm auf dem männlichen Theile des Knopfes befestigt. Die Grösse des Knopfes hindert den vorgezogenen Darm ebenso am Zurückschlüpfen, wie der Ring. Es kommen ebenso breite Berührungsflächen mit einander in Contact, die Schleimhaut des Aftertheiles, wenn sie stehen geblieben ist, wird ebenfalls exstirpirt. Von der sacralen Wunde aus werden 3-4 feste Seidennähte angelegt, die die beiden Darmrohre noch gesondert an einander befestigen.

Die Anlegung dieses grossen Murphyknopfes ist bequem und noch rascher ausführbar als die Naht des Darmes an dem Ring; für den grösseren Vortheil aber halte ich, dass durch diesen Verschluss eine Verunreinigung auch nur des Verbandes

1) Sieber, Ueber die Befestigung des Rectum nach Nicoladoni, ein Beitrag zur Technik der Mastdarmoperationen. Inaug.-Dissert. Breslau 1900.

2) Henle, Verhandlungen der Dentschen Gesellschaft f. Chirurgie. 1900. I. Theil. S. 34 . 
bei eintretendem dünnflüssigen Stuhle sicher vermieden wird und sowohl dieser, als auch die Darmgase naclr aussen abgeleitet werden. Das Rohr gestattet obne Verbandwechsel Darmeingiessungen. Wenn man die Knopfhälften nur mässig fest an einander presst, so hält der Knopf 7-8 Tage, dann fällt er mit dem Rohre ab, zu einer Zeit, da die Infectionsgefahr sowohl für die sacrale Wunde, als anch für das Peritoneum auf ein Minimum gesunken ist.

Ich hatte bisher dreimal Gelegenheit, so vorzugehen. Einmal bei einem 66 jährigen Manne mit hochsitzendem Rectum- und Flexurcarcinom, bei dem das Colon descendens abnorm beweglich war und das Durchziehen der oberen Hälfte der Flexur durch den anus gestattete. Ein zweites Mal handelte es sich um eine 44 jährige Frau mit einem ebenfalls hochsitzenden Mastdarmearcinom, wo ebenfalls die Flexur in ihrem untersten Antheile durch den Analring gezogen wurde und in oben angegebener Weise befestigt worden ist. Beide Fälle zeichneten sich durch einen besonders günstigen Heilungsverlauf aus. Beide Fälle wurden im September v. J. operirt. Bei der Frau betrug die Heilungsdauer kaum 4 Wochen; dies ist wohl dem Umstande zuzuschreiben, dass die sacrale Wunde im Vertrauen auf sichere Versorgung des Darmendes fast ganz geschlossen werden konnte und nicht wie gewöhnlich durch grosse und lange Streifen von Jodoform- oder steriler Gaze tamponirt wurde. 1 )

Möglichst breites und frühzeitiges Eröffnen des Peritonealcavum, reichlich bemessene Mobilisirung des Darmes mit gleichzeitiger möglichsten Schonung der Gefässe und Vermeidung irgend welcher Spannung: am Darme sind zum Gelingen unbedingt nöthig. Sehr lehrreich sind die Mittheilungen Kümmell's (l. c.) über das Durchziehen hoher Dickdarmabschnitte durch den Analring. Die Gefässversorgung wird umso besser, je weiter central man am Darme vordringt; die sogenannten Randgefässe, die sehr wichtige arterielle Anastomosen zwischen den eiuzelnen Hauptarterien des Dickdarmes darstellen, verzweigen sich (siehe Toldt's Atlas VI. Lfg. p. 579 fig. 955) a uch noch ein Stück weit in der Flexur. Die Durchtrennung des Mesenteriums an der Flexur, der Mesoflexur soll, wenn möglich, 3-4 cm vom Mesoflexuransatze an dem Darme entfernt sein, um jene

1) Seither verwendete ich einen Knopf, wie er in Fig. 1 abgebildet ist, in einem III. Falle bei einem 45 jähr. Manne und sind noch zwei weitere Mastdarmresectionen an der Grazer chirurgischen Klinik mit dieser Technik operirt worden, und hat dieselbe jedesmal vollständig befriedigt. 
Randgefässe zu schonen. Auf dieses Verhalten aufmerksam gemacht, habe ich die Ueberzeugung gewonnen, dass man bei möglichst weiter Eröffnung des Peritoneums und Herausziehen des Dickdarmes die nothwendigen Ligaturen zur Mobilisirung nur unter Berücksichtigung der Ernährungsverhältnisse machen soll, so dass man schon während der Operation sich über Lebenskraft des mobil gemachten Darmstückes klar ist.

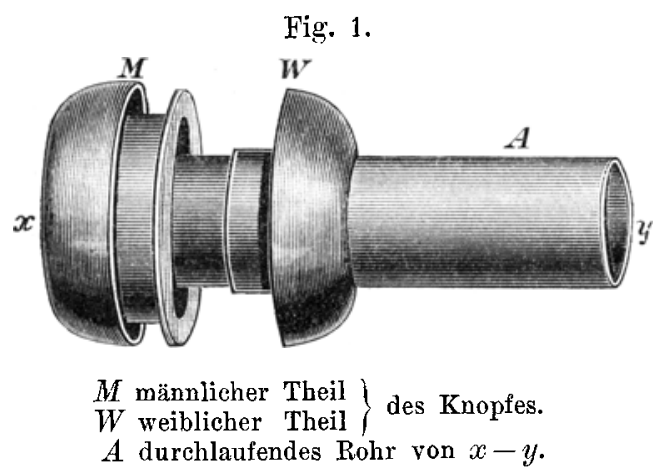

Fig. 1 stellt den neuerdings verbesserten Knopf in $3 / 4$ natürlicher Grösse dar; er hat keine seitlichen Löcher mehr und ist der männliche Theil M noch durch das ca. $7 \mathrm{~cm}$ lange Rohr A verlängert; der weibliche Theil, W wird daraufgeschoben. Durch M und das Rohr A wird der Darm hermetisch verschlossen; das Rohr A muss so gearbeitet sein, dass es keine seitliche Oeffnung enthält (z. B. für die Feder). Auf das Rohr A wird das Ableitungsrohr aufgesteckt; früher, bei den ersten Fällen verwendeten wir einen gewöhnlichen, aber extragrossen Murphyknopf, durch den das gefettete Drainrohr gesteckt und mit Nähten an den Löchern befestigt wurde.

Die beigegebene Figur 2 stellt die Lage des Knopfes im Darmrohre und das Verhältniss zum Sphincterantheile dar. Diese Technik ist ebenso gut bei der Amputatio recti möglich.

Heinecke (l. c.) hat den Sphincterantheil median gespalten; dies ist ein auch heute von vielen Chirurgen nachgeahmter Vorgang; die Spaltung erlaubt in höchst bequemer Weise die Exstirpation der Schleimhaut.

Hochenegg dehnt vor dem Akte des Herausziehens den Sphincter und hält dies für so wichtig, dass er sogar schlechtere Continenzverhältnisse dafür in Kauf nimmt.

In seinen ersten Publicationen theilt Hochenegg mit, dass er ein mit Jodoformgaze gut umwickeltes Drainrohr nach ge- 
machter Resectio recti vom Anus aus einschiebt. In seinen letzten Mittheilungen hören wir nichts von diesem "Stopfrohr", das wohl hauptsächlich den Zweck hatte, die damals gebräuchliche Circulärnaht vor dem Drucke und der Beschmutzung der Faekalmassen zu schützen.

Fig. 2.

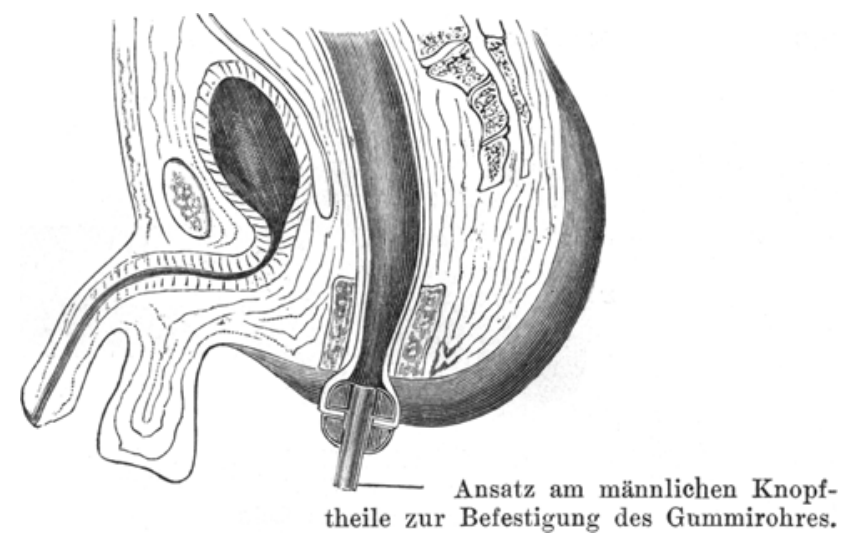

Unser Drainrohr, bei Verwendung eines gewöhnlichen Murphyknopfes, reicht höchstens $1 \frac{1 / 2}{2}-2 \mathrm{~cm}$ über den in den Darm sehenden Absehnitt des Murphyknopfes hinaus. Das Rohr ist durch 3-4 Seidennähte an die Löcher des Murphyknopfes befestigt und kann bei unbeabsichtigten Bewegungen und Lageveränderungen des Kranken nicht herausgezogen werden. Es ist etwa zeigefingerdick und muss so gewählt sein, dass es im Lumen des Knopfes festgeklemmt ist. In unsern ersten beiden Fällen kam in den ersten 8 Tagen niemals eine Beschmutzung des Verbandes mit Stuhlmassen vor, obwohl reichlich salinische Abführmittel gegeben wurden. Wie schon erwähnt, eignet sich ein etwas anders construirter Murphyknopf (Fig. 1) noch besser und ist der Abschluss dureh das auf das Rohr A aufgesteckte Rohr sicherer.

\section{Zur Technik der Colotomie und Colostomie.}

Eine ganze Reihe von Erkrankungen des Dickdarmes erheischen die Anlegung eines widernatürlichen Afters. Deñ Vorrang unter diesen nehmen wohl die inoperablen Careinome des Mastdarmes ein, oder jene Fälle, denen als Vorbereitungscur eine Colostomie vorausgeschickt wird. Daran reihen sich die so mannigfachen Verengungen des Mastdarmes durch luetisehe, gonorrhoische, dysenterisehe und andere chronisch entzündliche Processe u. s. w. Während diese 
geschilderten Erkrankungen durch die Palliativoperation des künstlichen Afters für kürzere oder längere Zeit in einen halbwegs erträglichen Zustand versetzt werden sollen, kommen noch Colostomieen dazu, die angelegt werden, um einen durch tiefe Darmstenose bedingten Ileus zu beseitigen $u$. s. w. Es sind Operationen, die meist sehr rasch ausgeführt werden müssen, weil der Organismus der bedauernswerthen Kranken bereits durch die andauernde Passagestörung im Darme und die dadurch bedingte Intoxication erschöpft ist.

2 Hauptgesichtspunkte sind es, die die technische Entwicklung dieser Operation bestimmten, einmal die möglichste Vermeidung jeder Infectionsgefahr, sei es für den Bauchraum, oder für die Bauchdecken. Die Bauchdeckenphlegmone war ebenfalls eine sehr gefürchtete Wundeomplication dieses Eingriffes.

In zweiter Linie suchte man durch die verschiedenartigsten Verfahren eine gewisse Schlussfähigkeit des gebildeten widernatürlichen Afters zu erzielen, um eine stäte Besudelung des Kranken durch die nachdrängenden Kothmassen thunlichst zu vermeiden.

Der ersten Indication genügte man wohl am besten durch das zweizeitige Op eriren. Doch giebt es eben Fälle, wo man nicht zwei, ja nicht einen Tag Zeit hat. Und diese Fälle der einzeitigen Operation wurden nicht selten auch in jüngster Zeit noch von Bauchdeckenphlegmonen in unangenehmster Weise gestört. Auch hier ist ein Verfahren am Platze, das möglichst die Reinlichkeit bei der Kothentleerung und die sichere Versorgung des eingenähten Darmantheiles garantirt. Für jene Fälle, wo es gilt, bei Ileus sehr rasch einen anus praeternaturalis anzulegen, ist eine möglichste Vereinfachung der Operationstechnik anzustreben.

Das Annähen eines alsogleich zu eröffnenden Darmantheiles muss gewisse Gefahren mit sich bringen; einerseits die Gefahr einer Infection der Bauchhöhle, andrerseits die einer Verunreinigung der Bauchdeckenwunde.

Der Gedanke, durch weites Hervorziehen eines Darmtheiles die Operationswunde und das Bauchfell vor Infection mit'Koth zu schïtzen, war schon fast vor 2 Jahrzehnten ausgesprochen und ausgeführt worden. Partsch1) hat bei Anlegung eines anus praeternaturalis am Dünndarme wegen eines stenosirenden Krebses ein rom Mesenterium abgelöstes Darmstück ein gutes Stiuck weit aus der Bauchwunde hervorgezogen, am Ende provisorisch durch Ligatur versehlossen, und durch Peritonealnähte umsäumt; dadurch wurde ein natürliches

1) Partsch, Laparotomie wegen Krebs des Dünndarmes. Centralblatt f. Chirurgie. 1883. Nr. 52. S. 833. 
Abzugssrohr für den Darminhalt geschaffen, das einen Schutz von Infection gewährte. Dieses Verfahren scheint nahezu vergessen worden zu sein.

Lauenstein ${ }^{1}$ ) veröffentlichte 11 Jahre nach Partsch's Operationsvorschlag ein Verfahren, das darin besteht, dass man bei der Anlegung eines dauernden anus praeternaturalis ein 15-20 cm langes Stïck des zuführenden Darmes durch die angelegte Wunde, eventuell unter Ablösung des Mesenteriums hervorzieht und dasselbe da, wo es durch die Bauchdecken tritt, durch Nähte fixirt. Es entsteht dadurch ein "penisartiges" Gebilde, durch das man einerseits den Koth in ein Gefäss mit Vermeidung von Verunreinigung ableiten kann, das andrerseits Gelegenheit giebt zur Anbringung der verschiedenartigst gedachten Verschluss-Apparate und Recipienten. Es ist in Lauensteins (l. c.) so operirtem Falle keinerlei Ernährungsstörung im vorgelagerten Darme aufgetreten. ${ }^{2}$ ) v. $\mathrm{Hacker}^{4}$ ) operirte in einem solchen Falle nach diesem Vorschlage, sah sich jedoch veranlasst, später den penisartigen Darmtheil abzutragen, und ist der Ansicht, dass sich Lauenstein's Vorgang nur dann gut durchführen liesse, wenn man den vorgezogenen Darm an der Serosafläche mit Haut überziehen würde, was ein immerhin complicirtes Verfahren ist.

Wir haben in der letzten Zeit eine Anzahl von Colotomieen und Colostomieen ausgeführt und dabei den Darm in einer sogleich zu erörternden Weise versorgt. Es handelte sich bei unsern Fällen theils um solche, wo wegen einer bestehenden Strictur am rectum die Colotomie als Palliativ-Operation gemacht worden ist und die Oeffnung des Darmes einem dauernden widernatürlichen After entsprach, und um durch Ileus-Erscheinungen bedingte rasch ausgeführte Eröfnung des Darmes oberhalb der verengten Stelle, unentschieden ob es späterbin zu einer Beseitigung des Afters komme oder nicht.

In einem derartigen Falle, es handelte sich um ein sehr arg stenosirendes, hochsitzendes Rectumcarcinom, wurde nach gemachter Laparotomie die Flexur hervorgezogen, quer durchtrennt, das periphere Stïck nach Anlegung einer doppelreihigen Verschliessungsnaht ${ }^{5}$ ) in

1) Lauenstein, Zur Colotomie. Centralbl. f. Chirurgie. 1889. Nr. 24. S. 439.

2) Auf einem ähnlichen Gedankengang beruht das vor Jahren von Rie del ${ }^{3}$ ) angegebene Verfahren bei Gangrän verdächtiger Darmschlingen bei eingeklemmtem Bruche, die Schlingen auch der Bauchhöhle hervorzuziehen und auf ein Verbandstïck zu lagern.

3) Riedel, Ueber Behandlung gangränöser Hernien. Deutsche med. Wochenschrift. 1883 . Nr. 45.

4) v. Hacker, Colostomie mit Sphincterbildung aus dem linken M. rectus abdominis. Beiträge zur klin. Chirurgie. Bd. XXIII. S. 628.

5) Für gewöhnlich empfiehlt es sich auch das periphere Stück nach der Durchtrennung offen zu lassen und in die Bauchdeckenwunde einzunähen. 
einen Winkel der Bauchwunde eingenäht, das centrale Ende aber provisorisch verschlossen und mit einigen wenigen Nähten an Peritoneum und Fascia transversa befestigt.

Nachdem dies geschehen war, wurde ebenso, wie wir es bei der Versorgung des proximalen Darmstückes nach der Entfernung des krebsigen Mastdarmes beschrieben haben, ein grosser Murphyknopf mit Drainrohr armirt, mit seinem männlichen Theile in das offene Darmlumen der Flexur eingeschoben, die vorher angelegte Tabaksbeutelnaht goschlossen und die weibliche Hälfte mässig fest auf die männliche gedrückt. Das eingefettete Drainrohr bewirkte einen völlig dichten Kothabschluss und konnte die Bauchdeckenwunde bis auf zwei kleine Jodoformgazestreifchen, die zu beiden Seiten des Murphyknopfes lagen, in Etagen geschlossen werden. Fünfeinhalb Tage hielt der Knopf, es wurden Eingiessungen durch das Drainrohr in den Darm gemacht und sowohl Darmgase, als festerer Inhalt durch das Rohr ohne jede Besudelung der Wunde nach aussen in eine Vorlage entleert. Die Heilung der Bauchdeckenwunde ging sehr rasch und anstandslos von statten. Die völlige Abtrennung des Darmes ist unseres Erachtens für das weitere Verhalten der dadurch vollkommen ausgeschalteten carcinomatösen Darmpartien von grösster Bedeutung. Wir haben in manchen Fällen in Uebereinstimmung mit vielen anderen Chirurgen zeitweilig beinahe einen Stillstand des Wachsthums beobachtet und war die Lebensdauer durch diese Eingriffe beträchtlich verlängert. Der betreffende Patient hat seit der Operation $(3 / 4 \mathrm{Jahr})$ um $8 \mathrm{~kg}$ zugenommen und ist wieder berufsfähig geworden.

Der Murphyknopf kann aber auch verwendet werden, um den Darm fest an Peritoneum und Fascia transversa - dieses Verfahren eignet sich besonders für Nothoperationen bei Ileus - oder auch an irgend eine andere Schichte der Bauchwand anzuheften. Handelt es sich um quere Durchtrennung der hervorgezogenen Schlingen, so genïgt eine kleine Oeffnung in der vorderen Bauchwand, um den Darm durchtreten zu lassen; der dicke, eingelegte Murphyknopf verhütet das Zurückgleiten des herausgezogenen Darmrohres und macht im Nothfalle die Umsäumungsnaht, die die Darmschlinge oder das Darmrohr an das Peritoneum parietale befestigt, überflüssig. Man kann durch Verwendung derartiger, grossgebauter Murphyknöpfe, die dicke Drainröhren exact schliessend passiren oder auf das Rohr A Fig. 1 aufstecken lassen, den hervorgeholten Darm, sowohl als Schlinge, an deren Convexität der Knopf angebracht wird, als auch als quer durchtrenntes Rohr mit der vorderen Bauchwand in festen Contakt bringen. Nach 5-6-8 Tagen lässt sich der Knopf 
durch leichten Zug an dem in ihm befestigten Gummidrain entfernen; stehen bleibende necrotische Gewebsstückchen werden mit Scheere und Pincette entfernt.

Wir haben dieses Verfahren mit verschiedenen möglichen Modificationen bei mehreren Colotomien und Colostomien ${ }^{1}$ ) angewendet und auch in den Fällen, in denen einzeitig operirt wurde, durch völliges Fernbleiben des Kothes von Peritoneum, Bauchdecken und $W$ unde in den ersten 5-6 Tagen jene Gefahren vermieden, die bei einzeitigem Vorgehen den Erfolg bedrohen. Für jene Fälle, in denen man sich begnügen muss, möglichst rasch einen anus praeternaturalis anzulegen, giebt es mit Hilfe des in Fig. 1 abgebildeten Knopfes ein noch viel einfacher zum Ziele führendes Verfahren:

Von einem kleinen Laparotomieschnitte aus wird das betreffende Darmstück (wohl meistens Dickdarm), vorgezogen, ausgestrichen, durch Fingerdruck oder Compressorien beiderseits abgesperrt, an der Convexität der Schlinge eröffnet, so dass der männliche Theil des Murphyknopfes eben passiren kann. Knotung der vorher angelegten Tabaksbeutelnaht, Verschluss des Rohres A durch feste Gummikappe oder festen Wattepfropf. - Neben oder etwas höher, als die zum Vorziehen der Schlinge gemachte Oeffnung, wird durch Fascia transversa und Peritoneum eine zweite kleine Oeffnung gemacht, die mit der Knopfhälfte MA armirte Schlinge für einen Augenblick reponirt und das Rohr A durch das kleine Loch in Peritoneum und Fascia transversa geschoben, so dass es in der Bauchdeckenwunde wieder erscheint. (Diese kleine Oeffnung ist am besten durch Einschneiden auf eine von innen her angedrängte Kornzange zu machen). Nun wird der weibliche Theil des Knopfes über das Rohr A geschoben und mässig fest zusammengedrückt.

Der Darm ist an das Peritoneum parietale und die Fascia trsv. durch den breiten Knopf angepresst und stehen ziemlich grosse Serosaflächen in Berührung.

Ein Vortheil dieses Knopfes ist ferner, dass man nach 2-3 Tagen seine beiden Hälften fester aneinander drücken kann.

Die Lage des Darmes, des Knopfes und der Bauchdecken ist aus Fig. 3 zu ersehen. - Der Laparotomieschnitt, durch den die Schlinge hervorgeholt wurde, kann nun völlig in Etagen geschlossen werden und bleibt nur in der Musculatur und Haut eine Oeffnung zurück, die gerade so gross, als die weibliche Knopflälfte ist. Zwei kleine Gaze-

1) In den letzten $3 / 4$ Jahren sind $i$ Colotomien und Colostomien an der Klinik nach dieser Technik gemacht worden. 
streifen werden zu beiden Seiten des Knopfes an die Fasc. transversa gelegt.

Dies Verfahren ist natürlich auch für jene Fälle brauchbar, wo man den Darm quer durchtrennt und das proximale Stück zur Afterbildung benützt.

Fig. 3 .

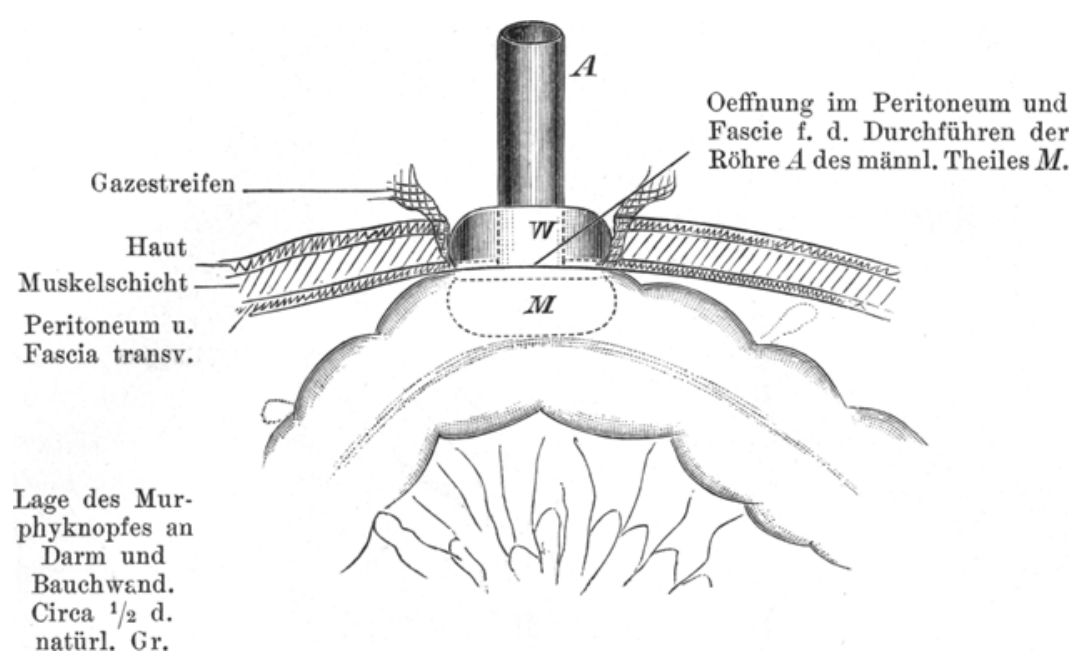

Wir halten in jenen Fällen, in denen sehr rasch operirt werden muss, die oben angegebene Methodik wegen ihrer Schnelligkeit, Einfachheit und Reinlichkeit für besonders empfehlenswerth. Wir erwähnen noch nebenbei, dass der Zeitpunkt des Durchschneidens des Murphyknopfes durch die Darmwand einerseits, das parietale Bauchfell und die Fascia transversa andrerseits durch die Stärke des Druckes beim Zusammenpressen der Knopfhälften annähernd bestimmbar ist und dass es sich empfiehlt, die Knopfhälften nicht allzu fest aneinander zu pressen, weil ein längeres Liegenbleiben des Knopfes in situ die Infectionsgefahr der Bauchdeckenwunde nur verringern kann.

Die Schleimhaut, die nach einiger Zeit vielleicht etwas prolabirt, kann eventuell noch secundär mit der äusseren Haut in Verbindung gebracht werden Ein solcher Art erzeugter künstlicher After entbehrt natürlich jeglicher organischen Verschliessungsvorrichtung und muss durch Pelottendruck oder anderweitige Bandagen geschlossen gehalten werden.

In allerletzter Zeit wurde der Plan gefasst, einen Verschluss bei den diesen besonders erheischenden Fällen auf folgende Art herzustellen: 
Es wird die Operation mit der Bildung eines zungenförmigen Hautlappens begonnen: für die Colostomie an der Flexura sigmoidea liegt die Längsachse dieses Lappens parallel zum linken Ligamentum Pouparti (Fig. 4). Die Länge des Lappens beträgt ca. $15-18 \mathrm{~cm}$, seine Breite $7-8 \mathrm{~cm}$. Der Lappen wird sofort nach seiner Abpräparirung gedoppelt, so dass eine aus einer zweifachen

Fig. 4.

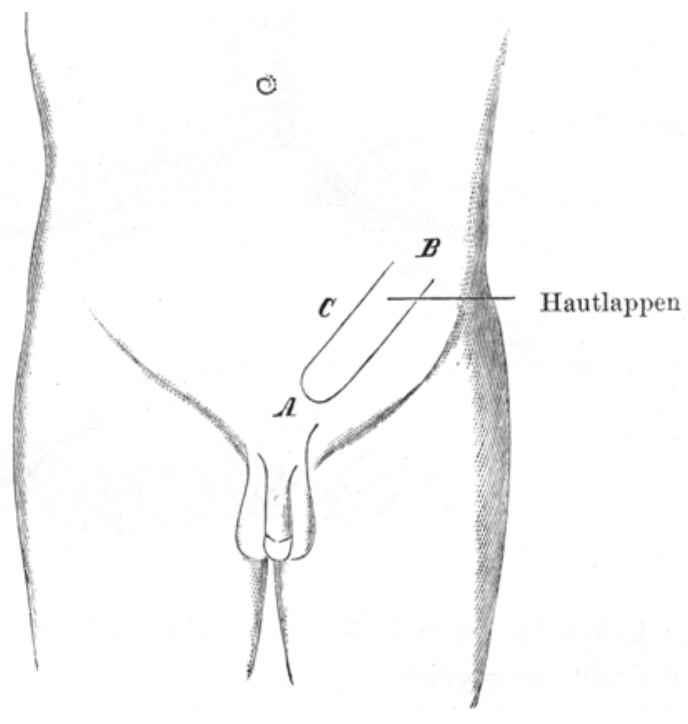

Lage von Haut bestehende Klappe von ca. $6-7 \mathrm{~cm}$ Länge und $5-6 \mathrm{~cm}$ Breite entsteht, wenn man die Wirkung der Elasticität der Haut auf die Dimensionen des Lappens berücksichtigt. Der Theil des Defectes, der durch den nunmehr gedoppelten Lappen nicht mehr bedeckt wird, wird allsogleich durch eine exacte Hautnaht linear vereinigt (Fig. 5 A-C).

Nun wird dem von der Hautklappe bedeckten Theile des Hautdefectes entsprechend die Bauchwand durchtrennt, die Flexurschlinge hervorgeholt und entweder abgetrennt oder über dem Jodoformgazestreifen vorgelagert. Die Umsäumung der vorgezogenen Schlinge an das Peritoneum parietale, die sich in diesem Falle wohl empfiehlt, soll so gemacht werden, dass ein ca. hühnereigrosser Abschnitt der Convexität der Schlinge frei zu Tage liegt.

Hier empfiehlt sich das zweizeitige Operiren; nach einigen Tagen, wenn die Schlinge fest mit der Bauchwand verklebt ist, öffnet man sie und näht gleich die Schleimhaut an die bereits granulirenden Hautränder des Defectes heraus; der gedoppelte Hautlappen legt sich 
wie eine Fallthüre auf die Darmlumina. Bei Durchtrennung der vorgezogenen Schlinge schliesst er nur das eine Lumen. Diese Hautdecke ist eine natürliche, nicht verschiebbare, lebende Pelotte, die nur durch eine gewöhnliche Bauchbinde angedrückt zu werden braucht, um den künstlichen After gut zu verschliessen (Fig. 5). ' Es ist an dieser Stelle am Platze, die Anschauungen und Bestrebungen kurz

Fig. 5.

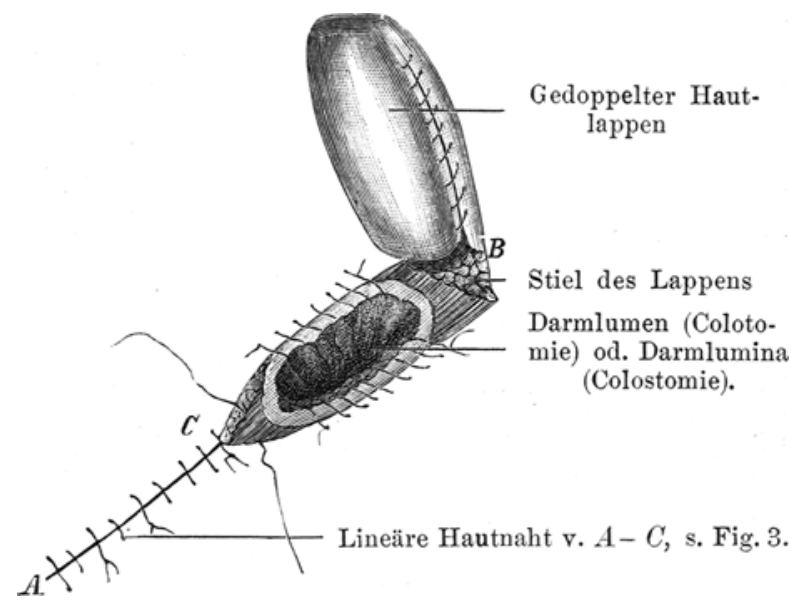

zu berücksichtigen, welche die Teehnik der Colostomie vom Standpunkte der Schlussfähigkeit in den letzten 15 Jahren geleitet hat.

Es sollte der künstliche After so beschaffen sein, dass fester Koth nur mit Willen des Patienten abgesetzt werden konnte, während man seit Jahren wusste, dass gegenüber flüssigem und halbflüssigem Darminhalte diese Bestrebungen nahezu fruchtlos waren.

Zwei Operationen am Magen-Darmkanale sind es vor allem, bei denen sich die Technik mit Aufwand aller Hilfsmittel bemüht hat, Verschluss - Vorrichtungen für künstliche Verbindungsgänge mit der Körperoberfläche zu erzielen. Bei der Gastrostomie und Jejunostomie kommt es darauf an, einen Kanal von der Körperoberfläche bis zum Verdauungsapparat herzustellen, der womöglich Flüssigkeiten den Eintritt von der Körperoberfläche zum Magen-Darmkanale, nicht aber den Rïicktritt solcher gestatten soll. Die Methoden, die dies zu erreichen suchen, setzen wir als völlig bekannt voraus.

Bei der Colostomie handelt es sich, eine genügend grosse Communication zwischen Dickdarm und Körperoberfläche herzustellen, die jedoch gewisse Hemmungsvorrichtungen besitzt, um ein fortwährendes Besudeltwerden der Umgebung des künstlichen Afters zu 
vermeiden, und bei geregelter Diät nur ein- oder zweimal des. Tages Kothmassen austreten lassen sollen. So genau diese Postulate theoretisch präcisirbar sind, so schwer sind sie in praxi zu erfüllen. Beweis dafür sind die zahlreichen Vorschläge zur Erreichung dieses Zieles. Doch ist bereits heute allgemein zugegeben, dass ein völlig befriedigender Verschlussmodus noch nicht gefunden ist und der Sphincter ani bislang durch keine auch noch so sinnreiche Methode einen Ersatz gefunden hat.

Czerny ${ }^{1}$ und viele andere ${ }^{2}$ ) sind daher auch in neuester Zeit bei der alten Littré'schen, von $\mathrm{Maydl}^{3}$ ) zweckmässig modificirten Methode der Colostomie geblieben und suchen durch Kautschukpelotten, die dem Darmlumen entsprechend gebaut sind, den Stuhl zurückzuhalten, was bei hartem Stuhle wohl gelingt.

Die Bemühungen jener, die durch operatives Vorgehen Verschlussvorrichtungen schaffen wollten, haben sehr verschiedene Wege eingeschlagen. Man liess den Darm einen längeren Weg unter einer Hautbrücke zurücklegen, um ihn daselbst bequem comprimiren zu können (Frank)4), man zog ihn durch ein Loch in der Darmbeinschaufel (Gleich) ${ }^{5}$, legte ihn in eine Rinne, die in die Symphyse geschnitten war $(\mathrm{R} \circ \mathrm{ux})^{6}$ ).

Des Vorschlages von Lauenstein (l. c.), ein längeres Darmstück zum Ableiten der Fäcalmassen zu benützen, haben wir bereits vorher Erwähnung gethan. Witzeli) und v. Hacker (l. c.) haben endlich in weiterer Nutzanwendung ihrer Methoden des Magenfistelverschlusses den M. rectus abdominis zur Bildung eines künstlichen Sphineters verwendet.

Ersterer zieht den Darm durch den in eine vordere und hintere

1) Czerny b. Lehmann, Ueber 45 Fülle von Colostomie an der Czerny schen Klinik. 1855-1895. Beiträge z. klin. Chirurgie. Bd. XVIII. S. 323.

2) Siehe auch Mikulicz, Handbuch der prakt. Chirurgie. Bd. III. S. 21 S.

3) Maydl, Zar Technik der Colotomie. Centralbl. f. Chirurgie. 1888. Nr. 24. S. 433 .

4) Frank, Neue Methode der Gastrostomie bei Carcinoma oesophagi. Wiener klin. Wochenschr. 1893. Nr. 13.

5) Gleich, Zur Technik des Anus praeternaturalis. Sitzungsbericht der Deutschen naturforschenden Gesellschaft. 1894. Centralbl. f. Chirurgie. 1894. S. 1086 .

6) Roux, C. von Mayer. Nouveau procêdé d'anus artificiel à la Clinique chirurgicale de Lausanne. Revue méd. de la Suisse rom. 1898. No. 1. Centralbl. f. Chirurgie. 1898. Nr. 39.

7) Witzel, Zur Indication und Technik der Colostomie und Enterostomic. Centralbl. f. Chirurgie. 1894. Nr. 40. 
Hälfte getheilten M. rectus durch, wobei gleichzeitig Pelottendruck zur Verbesserung des Verschlusses beiträgt; letzterer zieht von einem parallel zum Ligamentum Pouparti angelegten Schnitte aus die Flexur durch den in eine vordere und hintere Hälfte getrennten geraden Bauchmuskel durch und zieht sie ausserdem noch durch eine annähernd in der Mittellinie angelegte zweite kleine Hautwunde vor, so dass die vorgezogene Schlinge einen relativ sehr langen Weg zurücklegt, gedeckt durch den halbirten M. reetus und ausserdem noch durch eine Hautbrücke. Obwohl durch solche Bemühungen zweifellos bedeutend bessere Verschlüsse des künstlichen Afters erzielt worden sind, so ist es doch kaum zu erwarten, dass für halbflüssigen oder gar dünnflüssigen Darminhalt jemals eine halbwegs befriedigende Continenz geschaffen wird, und für dickbreiigen und festen Stuhl leisten die zahlreichen Pelottensysteme Befriedigendes.

Jene Methoden endlich, welche den Darm durch die Glutaealmusculatur durchtreten lassen, um einen Verschluss zu erzielen (Rydygier) 1), sind gegenwärtig wohl kaum mehr viel in Gebrauch. Da auf Reinigung von Seite des Patienten selbst soviel als möglich Rücksicht genommen werden sollte, so soll, wie dies Lauenstein schon ausgedrückt, der künstliche After an der Vorderseite angebracht werden. Die von Gersuny ${ }^{2}$ ) angegebene Drehung des Darmrohres um $180^{\circ}$ zur Erzielung einer Art von Verschlussklappe eignet sich nur für ein mit der Körperoberfläche in Communication zu bringendes Rohr, niemals aber für eine Schlinge. Die Pelotten liegen entweder glatt der Oeffnung an, oder sind gegen die Darmöffnung convex gebaut, bisweilen genau nach dem Lumen modellirt, oder endlich sie stïlpen sich wie eine Kappe über die Oeffnung, raumgebend für aufsaugende Verbandstoffe, Watte $\mathbf{u}$. dergl. Durch solche Vorrichtungen wird oft ein sehr guter Abschluss erzielt. Doch ist das gleichmässige, unverrückte Andrängen einer Pelotte an ganz bestimmter Stelle, wie sie auch gestaltet sein mag, etwas sehr schwieriges.

Ausgehend von dem Gedanken, eine nicht versehiebbare, lebende Pelotte zu bilden, die durch eine Bauchbinde an das zu verschliessende Darmlumen angepasst wird, und die auch bei den verschiedensten Körperbewegungen sich nicht verschiebt, kam ich zur Verwendung eines ganz gedoppelten Hautlappens, der auch einen gewissen Grad von Festigkeit besitzt und nicht etwa schlaff herabhängt.

1) Rydygier, Zur Bildung eines schlussfähigen Sphincter ani. Centralbl. f. Chirurgie. 1894. Nr. 4 ó.

2) Gersuny, Eine Sphincterplastik am Darm. Centralbl. f. Chirurgie. 1893. Nr. 26. 
Die glatte Oberfläche der Haut garantirt ein viel dichteres Anpassen an die Umgebung des Darmlumens, als die beste künstliche Leder- und Kautschukpelotte. Der fortwährende leichte Druck verhindert das sonst so oft beobachtete übermässig starke Prolabiren der Schleimhaut. Die Reinigung ist gewiss nicht schwieriger als bei irgend einer der übrigen Methoden, eher leichter, und wird, wenn, wie ich es mir vorstelle, ein Wattebausch zwischen die Bauchbinde und den Hautdeckel gelegt wird, die Bandage viel weniger beschmutzt, als bei den direkten Pelottenverschlüssen des künstlichen Afters. Der Eingriff ist technisch sehr einfach und leicht auszuführen, indem er sich von der gewöhnlichen Maydl'schen Colostomie eigentlich nur durch die Bildung des gedoppelten Hautlappens und die dabei nothwendigen Nähte im Lappen unterscheidet. Auch die Heilungsdauer dürfte kaum länger sein, da bei zweizeitigem Operiren schon nach ว-6 Tagen bei Eröffnung des Darmes die Infectionsgefahr für den Lappen und seine Wundverhältnisse eine geringe ist; für die einzeitig auszuführenden Colostomieen empfiehlt sich unseres Erachtens das Verfahren nicht sehr, da, wenn nach einer der früheren Methoden operirt wird, die Hautnähte leicht inficirt werden können; in einem solchen Falle müsste man die Eröffnung der vorgezogenen Schlinge oder Querdurchtrennung des Darmes sehr sorgfältig mit dem Murphyknopf und Drainrohr ausführen und durch möglichst langes Liegenlassen desselben die Reinlichkeit thunlichst zu bewabren suchen. Mögen diese Angaben und Vorschläge, die vielleicht doch für manche Fälle eine Erleichterung der Technik bedeuten, sich brauchbar und nützlich erweisen.

Wir legen ganz besonderes Gewicht darauf, dass die Versorgung des Darmendes, sei es auch zu welchem Zwecke, wenigstens soweit mit Reinhaltung der Wunde und des Verbandes geschehen kann, dass auch bei einzeitigem Operiren die Gefahr der Bauchdeckenphlegmone, Wundinfection, oder gar einer - heutzutage wohl kaum mehr vorkommenden - Peritonitis, minimal ist, wie mehrere nach diesen Vorschlägen ausgeführte Colostomieen und Resectionen des Rectums nach sacraler Methode uns bewiesen haben.

Was einen idealen Verschluss von absichtlich angelegten Communicationen zwischen Darmkanal und Körperoberfläche anlangt, so sind wir der Ansicht, dass diese Frage noch nicht zu einem Abschlusse gekommen ist, sondern die verschiedenartigsten Versuche, zu besseren Resultaten zu kommen, gerechtfertigt sind.

So sind wir der Ansicht, dass die Mittheilungen Kümmell's (l. c.) über seine so ausgedehnten Resectionen am Dickdarme mit Durch- 
ziehen des Colon transversum durch den Analring einen Fingerzeig: für weitere Bestrebungen geben.

Für gewisse, einen grossen Theil des Rectum freilassenden Carcinome wird ab und zu die Sigmoideo-Rectostomie (Rotter 1) in Frage kommen.

1. Durch die Verwendung grosser besonders gebauter Murphyknöpfe ${ }^{2}$ ), ist eine bequeme Versorgung des proximalen Darmendes bei Resectio und Amputatio recti möglich.

Die Vortheile des Verfahrens sind: Schnelligkeit, Sicherheit gegen das Zurückgleiten des durch die Analportion durehgezogenen Darmes, Gewähr möglichster Reinlich keit.

2. Solche Knöpfe eignen sich auch für Colostomieen oder als Palliativoperationen dienende Colotomieen; Verhütung yon peritonealen und Bauchdeckeninfectionen und grosse Reinlichkeit sind auch hier das Wesentliche.

3. Das Verfahren ist auch für jene Methoden der Darmfistelbildung geeignet, welche einen organischen Verschluss des Kunstafters beabsichtigen, wie Gersuny's Drehung, die Verfahren von v. Hacker und Witzel etc.

4. Für möglichst rasch anzulegende Colostomieen bei Darmverschluss bringen obige Knöpfe durch die Einfachheit und Raschheit der Ausführung bedingte Vortheile.

1) Rotter, Die Sigmoideo-Rectostomie, ein neues Verfahren zur Beseitigung von Mastdarmstricturen. Langenbeck's Archiv. Bd. LVIII. Heft 2.

2) Im Supplementhefte des XXIX. Bd. der Beiträge z. klin. Chirurgie lese ich im Vorworte v. Czerny von Friedrich'schen Knöpfen für Dickdarmoperationen; Herr Prof. Marwedel war so gütig, auf meine Anfrage mir mitzutheilen, dass eine anderweitige Beschreibung derselben noch nicht erfolgt sei; sie wurden bisher zu axialen Vereinigungen am Dickdarm verwendet. 